\title{
Hi in Early-type Galaxies
}

\author{
Tom Oosterloo ${ }^{1}$, Raffaella Morganti² and Elaine Sadler ${ }^{3}$ \\ ${ }^{1}$ Istituto di Fisica Cosmica, CNR, Via Bassini 15, 20133 Milan, Italy \\ toosterl@ifctr.mi.cnr.it \\ 2 Istituto di Radioastronomia, CNR, Via Gobetti 101, 40129 Bologna, Italy \\ rmorgant@ira.bo.cnr.it \\ 3 School of Physics, University of Sydney, NSW 2006, Australia \\ ems@physics.usyd.edu.au \\ Received 1998 November 2, accepted 1999 February 3
}

\begin{abstract}
We summarise the $\mathrm{H}_{\mathrm{I}}$ properties of early-type galaxies, in particular the differences in $\mathrm{HI}_{\mathrm{r}}$ morphologies observed in early-type galaxies of different luminosities. We find that in low-luminosity early-type galaxies the $\mathrm{HI}_{\mathrm{I}}$ is almost always in a disk-like structure, with central surface densities high enough for star formation to occur. In a few luminous early-type galaxies the $\mathrm{H}_{\mathrm{I}}$ is also in a disk or in a ring-like structure, but in most luminous early-type galaxies the $\mathrm{H}_{\mathrm{i}}$ has a relatively irregular morphology. The surface densities in the $\mathrm{H}_{\mathrm{I}}$ disks in luminous early-type galaxies are lower than in the $\mathrm{Hi}$ disks in low-luminosity early-type galaxies and no large-scale star formation should occur in these disks. We discuss these different $\mathrm{HI}$ characteristics in the context of other properties of early-type galaxies that correlate with luminosity.
\end{abstract}

Keywords: galaxies: elliptical and lenticular, cD-galaxies: ISM

\section{Introduction}

Early-type galaxies constitute quite a heterogeneous group of galaxies. Many of the properties of these systems vary from galaxy to galaxy and, more interestingly, vary systematically with luminosity and environment. Many of the differences may be related to the amount of gas (and dissipation) present during the formation and the evolution of the galaxies. Moreover, evidence is accumulating now that some early-type galaxies probably have a long-lived interstellar medium (ISM; e.g. Knapp 1998). To help understand the mechanisms and processes behind the differences between different early-type galaxies and to study the ISM observed in some of these galaxies, it may be worthwhile to study the systematics of the properties of the neutral hydrogen in early-type galaxies as a function of luminosity and environment. To this end, we have observed a large number of early-type galaxies with the Australia Telescope Compact Array and the Very Large Array (Morganti et al. 1997a,b, $1998 a, b)$. In particular, we have tried to observe galaxies over a range of luminosities in order to see whether the HI properties of low-luminosity early-type galaxies (which we define here as galaxies with absolute blue magnitude in the range of -16 to $-19)$ vary systematically with luminosity. Here we give a brief overview of the results obtained so far. In Section 2 we give a brief overview of properties of early-type galaxies observed at other wavebands that are relevant for the discussion. In Section 3 we briefly discuss the Hi content of early-type galaxies and in Section 4 we summarise the morphology and the kinematics of the HI as function of luminosity and environment and the relation of these properties to other properties of the galaxies.

\section{Properties at Other Wavelengths}

There are several properties of early-type galaxies that systematically vary with luminosity:

Stellar rotation. Some early-type galaxies are 'pressure supported', while in other galaxies the rotation of the stellar component is important for the dynamics of the system. In general, in luminous galaxies the random motions dominate while in lower luminosity systems the stellar rotation becomes more important (e.g. Davies et al. 1982).

Isophotal shape. In many early-type galaxies, the isophotes are not perfect ellipses. In lower luminosity galaxies, the isophotes are more often disky, while in luminous systems they tend to be more often box shaped.

Core properties. Imaging studies performed with HST have shown that the central density distributions vary systematically with luminosity (e.g. Lauer 1997). Low-luminosity systems usually have steeper cores that more luminous systems.

Excitation of the ionised gas. Many early-type galaxies have optical emission lines in their spectrum. The character of these lines changes systematically with luminosity. In low-luminosity systems, the spectrum is usually that of Hir regions. In luminous 
galaxies it corresponds to a liner spectrum (Sadler 1987). This indicates that the ionisation mechanism is different in these two types of galaxies.

Star formation history. The star formation history appears to change systematically with luminosity. For example, the relative abundance of $\mathrm{Mg}$ with respect to $\mathrm{Fe}$ correlates with velocity dispersion. Luminous galaxies typically have $[\mathrm{Mg} / \mathrm{Fe}] \sim 0 \cdot 4$, while fainter galaxies have values around 0 . This indicates that the enrichment history of the ISM changes systematically with luminosity. Lowerluminosity galaxies also show a larger spread in the Mg- $\sigma$ relation (e.g. Bender 1996), again pointing to a different star formation history. Many lowluminosity ellipticals in fact display star formation in the central parts of the galaxies. It appears that disky galaxies have stronger $\mathrm{H} \beta$ indices, indicating that some star formation occurred recently (de Jong \& Davies 1997).

$X$-ray emission. The amount of X-ray emission correlates strongly with optical luminosity. In large ellipticals, part of the X-ray emission originates from a halo of hot gas, while in smaller ellipticals the $\mathrm{X}$-ray is due only to X-ray binaries (e.g. Canizares et al. 1987).

Many of these differences between different galaxies can be explained by different amounts of gas present in the formation/evolution, and in many models for galaxy formation the gas supply is a key factor (e.g. Kauffmann 1996). For example, the differences between boxy and disky galaxies, the importance of rotation versus anisotropic galaxies, and the different central density distributions are possibly a consequence of the relative importance of gas. Obviously, since stars form from gas, the different star formation histories must be related to different gas contents during the evolution.

Considering the relation between gas and these different properties, it is worthwhile to investigate the systematics of the Hi properties of early-type galaxies.

\section{Hi Content}

Before discussing the Hi properties of early-type galaxies, it is important to define which type of galaxy is considered and in which environment the galaxies are. Table 1 lists the detection rates for different kinds of early-type galaxies. A few things are evident from this table. First, only a small fraction of 'pure' elliptical galaxies have detectable amounts of Hi. But as soon as the optical morphology shows some peculiarity, the probability of detecting Hi increases dramatically. This result has often been interpreted to mean that the origin of the $\mathrm{HI}$ in elliptical galaxies is external (e.g. Knapp, Turner \& Cunniffe 1985). It implies that if we investigate the characteristics of the $\mathrm{HI}_{\mathrm{I}}$ in these galaxies (morphology and kinematics), one is considering a subset of the whole population of early-type galaxies, namely those for which it is likely that some interaction/accretion in the recent past has occurred, and it is important to keep this in mind. Nevertheless it is important not to restrict samples to 'pure' ellipticals with no optical peculiarities, since the Hi-rich galaxies may represent an important phase in the evolution of many early-type galaxies.

Table 1. HI detection rates for early-type galaxies (from Bregman et al. 1992)

\begin{tabular}{lccr}
\hline Galaxy type & Observed & Detected & $\%$ \\
\hline E & 64 & 3 & 5 \\
E/S0 & 23 & 4 & 17 \\
S0, SB0 & 103 & 21 & 20 \\
Pec E and S0 & 20 & 9 & 45 \\
S0/a and pec & 35 & 15 & 43 \\
Sa and pec & 103 & 78 & 76 \\
\hline
\end{tabular}

Table 1 does however suggest that there may be a second origin for the Hi in early-type galaxies. The detection rate also depends strongly on how much stellar disk is present in a galaxy. This could imply that the presence of Hi in early-type galaxies is not always due to a recent accretion. The fact that the $\mathrm{HI}$ content is related to the fundamental structure of a galaxy could suggest that some of the disky galaxies may have a long-lived ISM. It appears that many early-type galaxies, especially those in the field, indeed often have an ISM with similar characteristics as the ISM in spirals, the main difference being that early-type galaxies have less of it (see e.g. Knapp 1998).

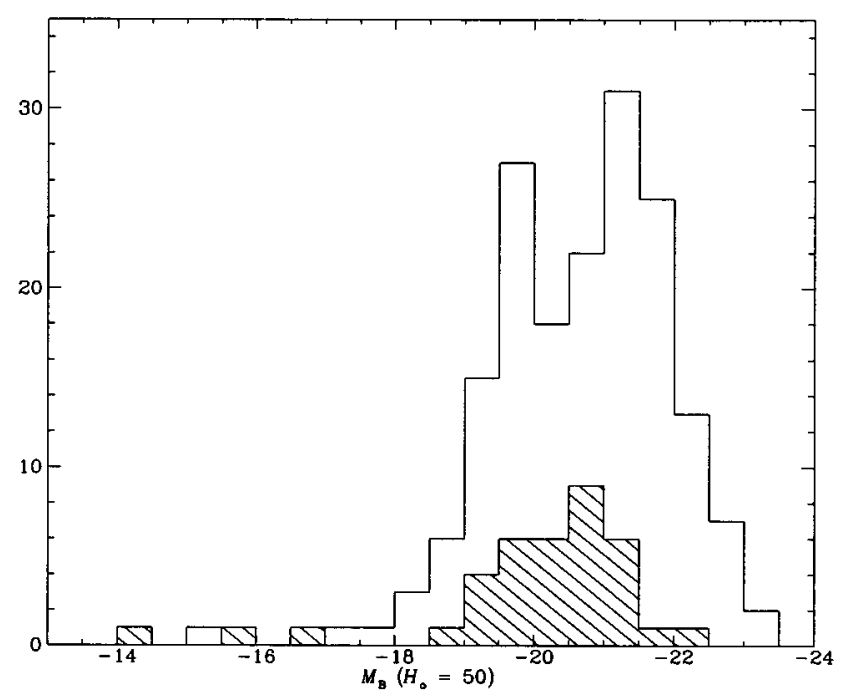

Figure 1- Hi detection rate of $\mathrm{E}$ and $\mathrm{E} / \mathrm{S} 0$ galaxies as function of absolute magnitude. The shaded histogram gives the distribution of the detections, and the unshaded histogram the distribution of the non-detections. Data are from Bregman, Hogg \& Roberts (1992). 


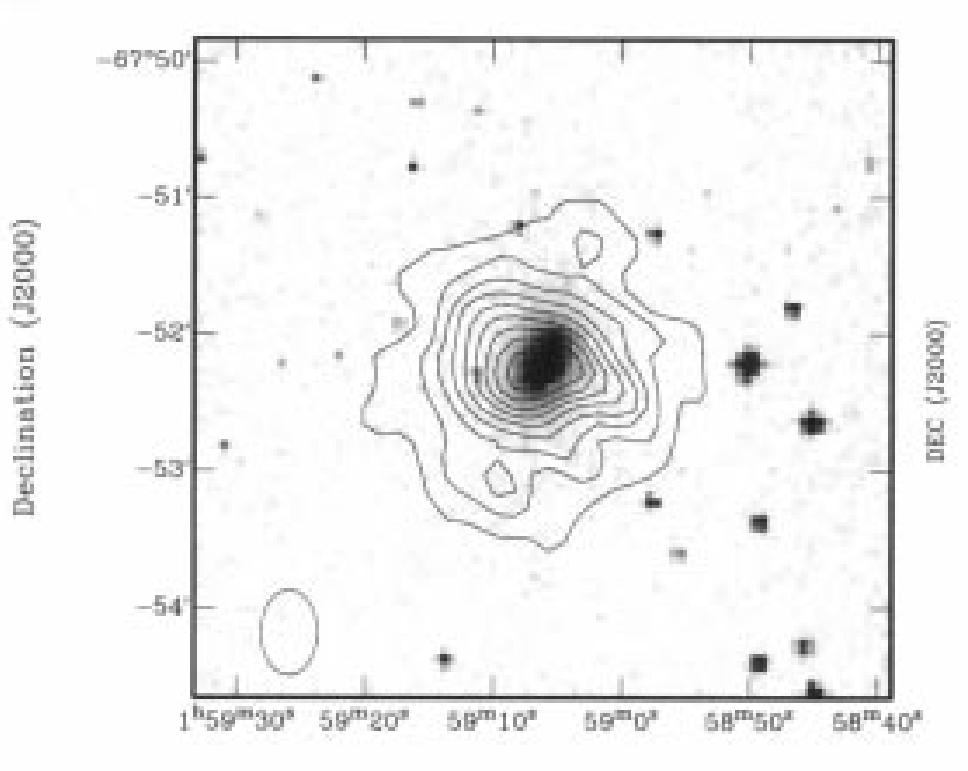

Right Ascension (J2000)

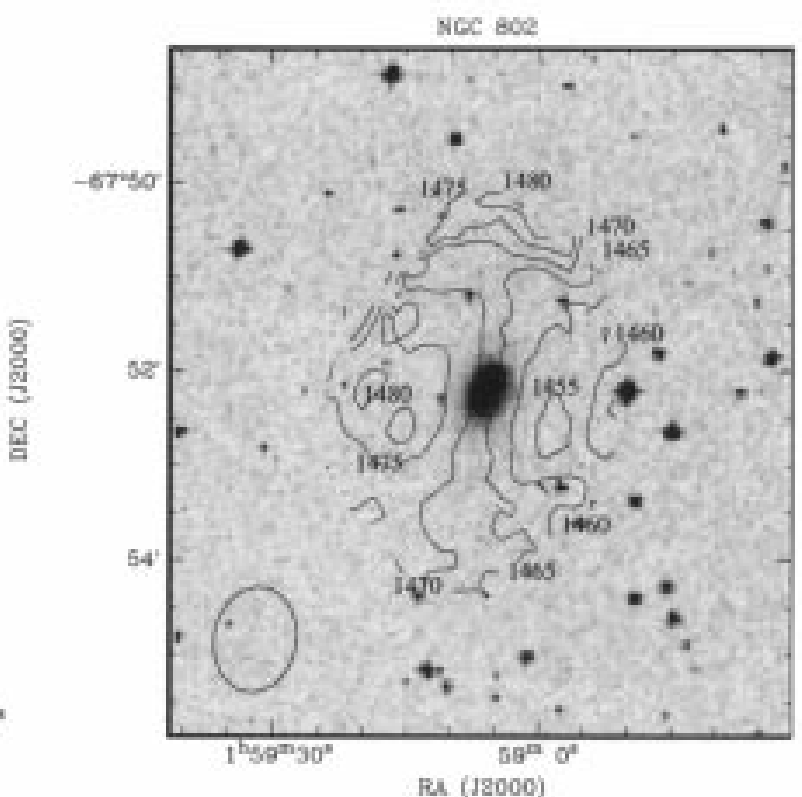

Ru $(12000)$

Figure 2-Left: Contours of the total Hi image of NGC 802 as obtained with the ATCA using uniform weighting. Right: HI velocity field of NGC 802 from the natural weighted ATCA data. Contour values (heliocentric, $\mathrm{km} \mathrm{s}^{-1}$ ) are indicated. The optical image in both figures is taken from the Digital Sky Survey.
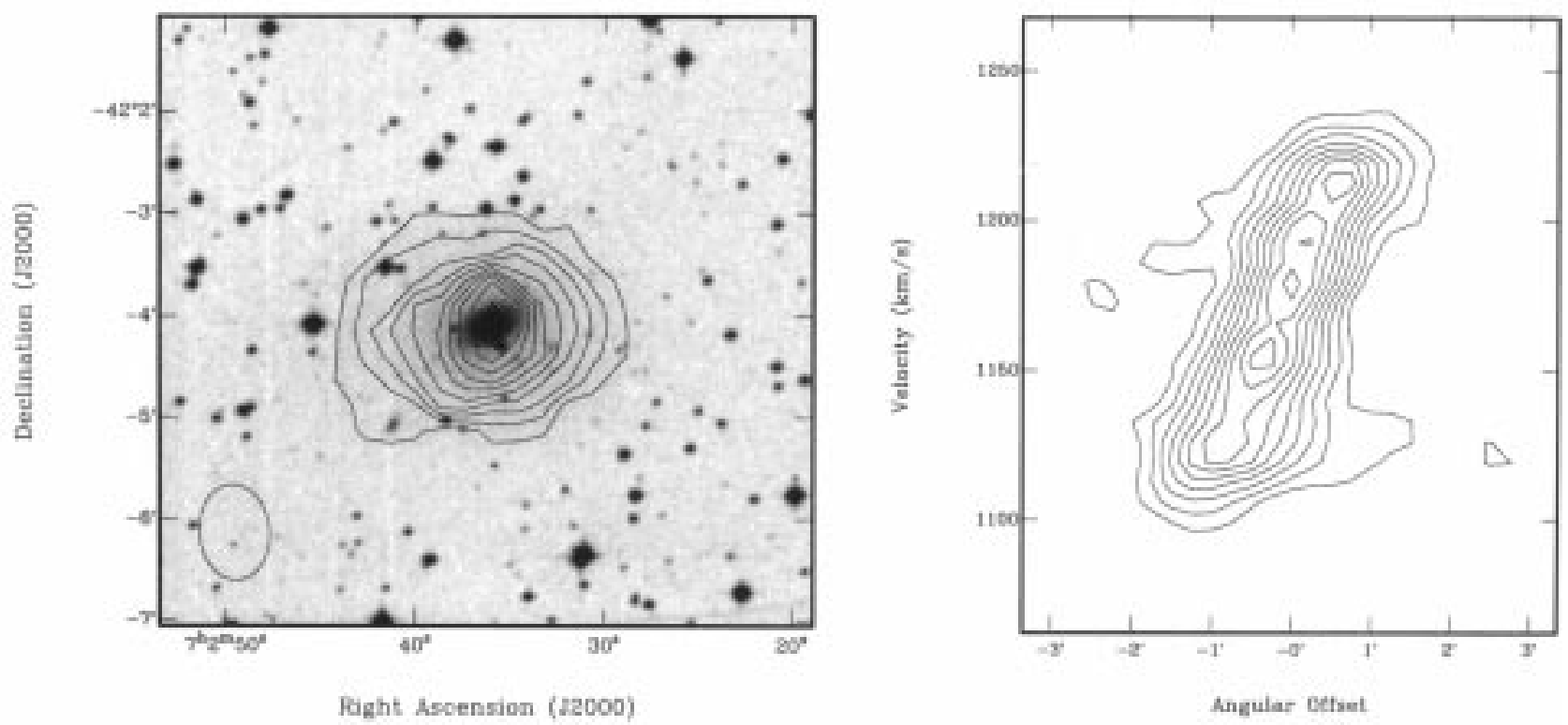

Figure 3-Left: Total Hi image of NGC 2328 as obtained with the ATCA. The optical image is taken from the Digital Sky Survey. Right: Position-velocity map taken along the major axis of NGC 2328.

It is often stated that low-luminosity early-type galaxies are more likely to have Hi. This is usually based on a study done by Lake and Schommer (1984) who observed a small sample of low-luminosity earlytype galaxies and found that the detection rate of their sample was significantly higher than that of more luminous early-type galaxies as it was known at the time. However, when using larger samples of $\mathrm{HI}$ data on early-type galaxies that are available now, the situation appears to be somewhat different from that suggested by Lake and Schommer.
In Figure 1 we show the detection rates of early-type galaxies as function of luminosity as it can be derived from the compilation of data of Bregman, Hogg \& Roberts (1992). The figure shows that galaxies brighter than absolute magnitude -22 appear to be poorer in Hi than galaxies fainter than this limit. However, for galaxies in the magnitude range -16 to $-21 \cdot 5$, the detection rate appears to be reasonably flat. There is no strong evidence that low-luminosity galaxies (absolute magnitude between -16 and -19) are richer in $\mathrm{HI}$ than galaxies in 


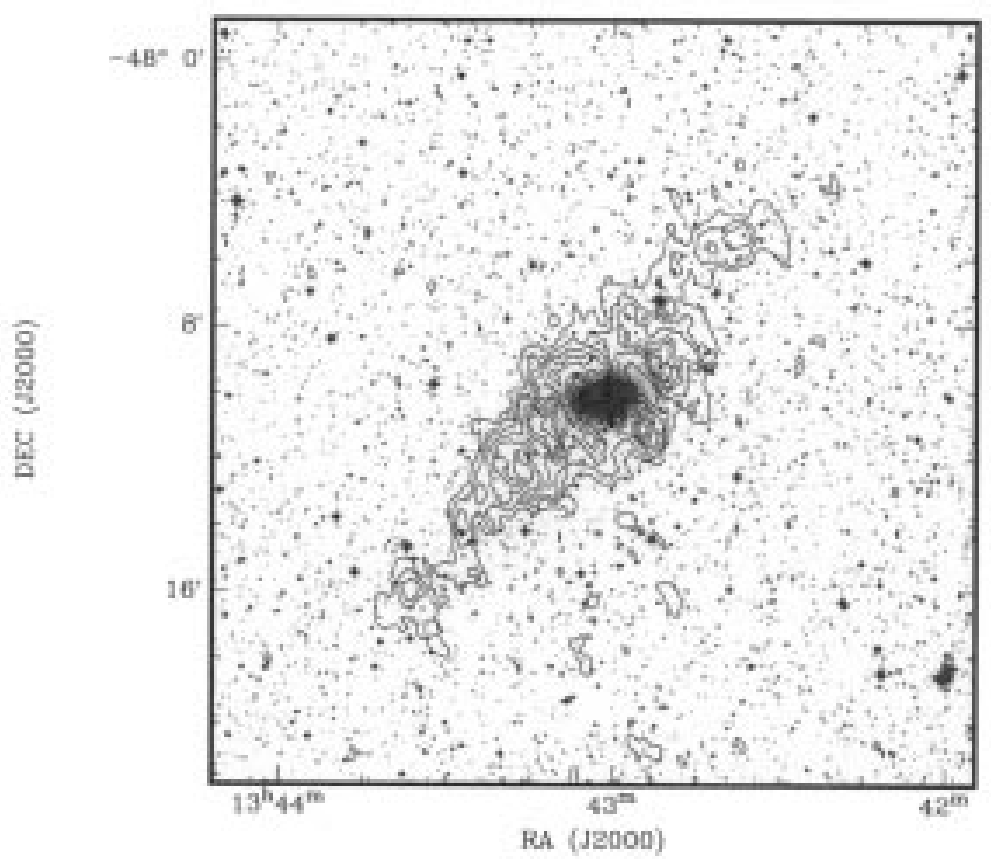

Figure 4-Contours of the total Hi image of NGC 5266 superposed on an optical image obtained from the Digital Sky Survey.

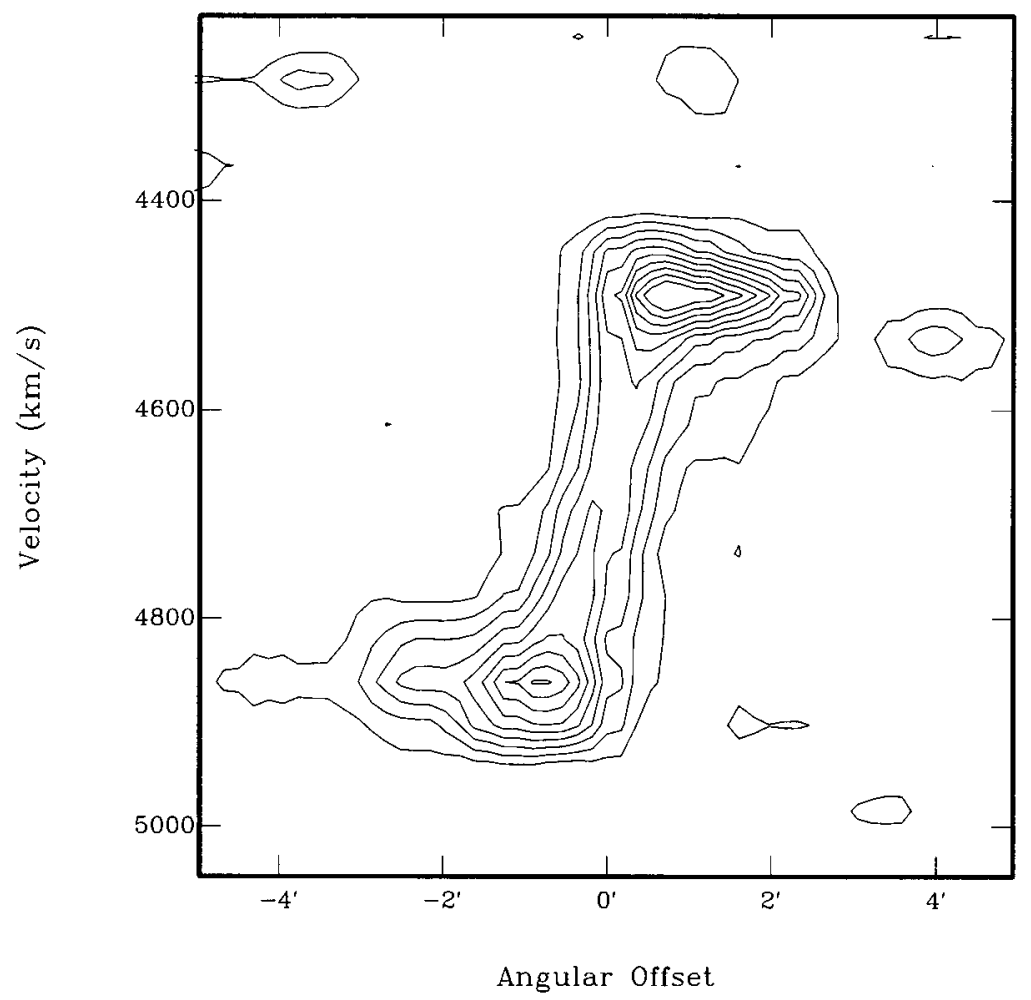

Figure 5-Position-velocity plot of NGC 807, taken along the kinematical major axis (data taken from the VLA archive).

the range -19 to -22 . A similar conclusion was obtained by Knapp, Turner \& Cunniffe (1985). It appears that a more correct statement about $\mathrm{HI}$ content would be that the most luminous galaxies are poor in Hi. Galaxies fainter than $M_{B}=-16$ appear to have more Hi, although the number of galaxies for which data are available is small. It is however, somewhat difficult to derive strong conclusions from the compilation of Bregman et al., because it consists of a mix of field and cluster 

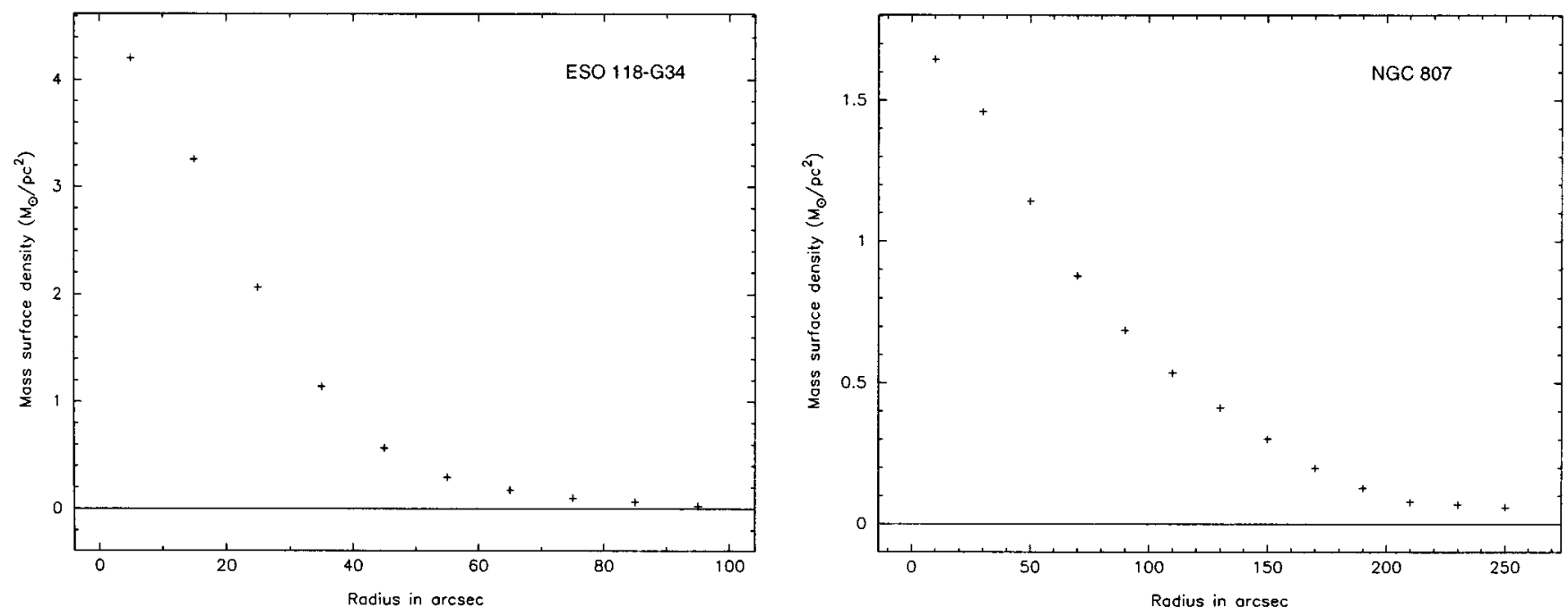

Figure 6-Radial Hi surface density profiles of the low-luminosity elliptical ESO 118-G34 (left) and the luminous E4 galaxy NGC 807 (right).

galaxies, and differential environmental effects are possibly important.

\section{Hi Morphology and Kinematics}

One interesting difference between low-luminosity galaxies $\left(-19>M_{B}>-16\right)$ compared to more luminous galaxies $\left(-22>M_{B}>-19\right)$ is that the range of morphology and kinematics observed is different for the two groups. From our data, together with data in the literature (e.g. Lake, Schommer \& van Gorkom 1987), there are now about $10 \mathrm{HI}$ data cubes available for low-luminosity early-type galaxies. Almost without exception, the Hi in these galaxies is in a disk with a regular morphology and kinematics. In some galaxies there is evidence that (part of) the Hi may have been accreted recently, but in several galaxies the structure of the $\mathrm{HI}$ is very regular and there is no evidence from the kinematics that a recent accretion has occurred. To illustrate this, in Figure 2 we give the total $\mathrm{HI}$ image and the velocity field for the galaxy NGC 802 $\left(M_{B}=-18\right)$. The Hi in this galaxy is very centrally concentrated. The velocity field shows however that the gas is rotating around the optical major axis, like in polar-ring galaxies. This suggests that the HI in NGC 802 has been accreted after the main stellar body had formed. Another low-luminosity galaxy that shows a very similar Hi configuration is NGC 855 (Walsh et al. 1990).

An example of a regular Hi disk in a low-luminosity galaxy is given in Figure 3, where we show the total Hi image and a position-velocity map taken along the major axis NGC 2328. Also in this galaxy the $\mathrm{HI}$ is quite centrally concentrated. The position-velocity map in Figure 3 shows that the HI in NGC 2328 is in a regularly rotating disk, aligned with the optical body.
In contrast to the low-luminosity galaxies, the range in $\mathrm{H}_{\mathrm{I}}$ morphology in the more luminous galaxies $\left(-22>M_{B}>-19\right)$ is much broader. For this group, in most galaxies the Hi shows an irregular morphology, indicating that the gas is accreting onto the galaxy, or is left over from a recent merger event. A good example of this is NGC 5266 (Figure 4; see Morganti et al. 1997a). This is a minor-axis dust-lane elliptical with a large amount of $\mathrm{HI}_{\mathrm{I}}\left(\sim 10^{10} M_{\odot}, M_{\mathrm{HI}} / L_{B} \sim 0 \cdot 2\right)$. Almost all the $\mathrm{HI}$ is in an elongated structure parallel to the optical major axis. Most of this gas is rotating in a reasonably regular fashion, although several subsystems can be identified that are not in stable circular rotation. Interestingly, this large-scale $\mathrm{HI}$ structure is perpendicular to the inner, minor axis, dust lane, and some Hi associated with this dust lane is also detected (a few percent of the Hi mass). Clearly, NGC 5266 is a system where a large amount of $\mathrm{Hi}_{\mathrm{I}}$ has been accreted recently, or is a remnant of a recent merger, and the $\mathrm{H}_{\mathrm{I}}$ is still settling in the galaxy.

Interestingly, there are now a few luminous earlytype galaxies known that do have very regular $\mathrm{HI}_{\mathrm{I}}$ structures. A very good example is the E4 galaxy NGC 807 (Figure 5). Deep Hi observations reveal a low-surface brightness $\mathrm{HI}$ disk that shows no sign that this Hi has been accreted recently. Figure 5 gives the position-velocity map of this Hi disk, clearly showing the regular rotation of this disk. The evolution of this disk is very slow, and this HI disk can be quite old. Often, these regular $\mathrm{HI}_{\mathrm{I}}$ structures have a depression or hole in the centre that is filled up with a disk of ionised gas that has very similar kinematics as the $\mathrm{HI}$ disk. A good example of this is the dust-lane galaxy NGC 3108 .

Another striking difference between the Hi structures seen in low-luminosity early-type galaxies, 
and in more luminous galaxies, is that the central surface brightnesses are quite different. In the low-luminosity galaxies, the $\mathrm{HI}_{\mathrm{I}}$ is quite centrally concentrated with central Hi surface densities of at least $4 M_{\odot} \mathrm{pc}^{-2}$. These densities are high enough for star formation to occur on a reasonable large scale, and indeed star formation is observed in the centres of these galaxies. Outside the centre, the surface densities of the $\mathrm{H}_{\mathrm{I}}$ are below $1 M_{\odot} \mathrm{pc}^{-2}$ and perhaps only sporadic star formation could occur. In contrast, the surface densities in the more luminous galaxies are much lower, even in the galaxies with a regular Hi disk or ring. The peak surface densities are typically around $1 M_{\odot} \mathrm{pc}^{-2}$, too low for large scale star formation to occur. Figure 6 shows the Hi density profile of a low-luminosity elliptical and of a more luminous E4 galaxy. The difference between these profiles is quite typical for what is observed in most galaxies.

\section{Connection with Other Properties}

The range of $\mathrm{HI}$ properties observed in early-type galaxies is quite large, but it appears that there are a few systematic trends in the data, and in particular that some of the Hi properties may be connected to properties observed at other wavebands.

Many low-luminosity early-type galaxies that have $\mathrm{HI}$, have this $\mathrm{HI}$ in a regularly rotating disk. In the optical, these galaxies also show a disky morphology and are rotationally supported. One possibility is that the Hi disk observed is the normal gas counterpart of the stellar disk structure in these galaxies. The central surface densities of the Hi are high enough for star formation to occur, and indeed star formation is observed in the centres of many of these galaxies, and the optical spectrum of the emission lines is that of HII regions. The higher densities of the Hi could also be related to the steeper cores that are observed in low-luminosity galaxies, although other mechanisms could be responsible for that. It appears that the Hi properties of low-luminosity early-type galaxies fit in with other properties of these galaxies, indicating that the ISM in these galaxies has played an important role in determining the structure of these galaxies. The regular Hi disks/rings observed in the more luminous galaxies could be similar in origin and character to the ones observed in the smaller galaxies (e.g. Morganti et al. 1998b), except that some mechanism must be responsible for keeping the central surface density of the Hi low. A clue to this mechanism could be that the centres of these regular structures are often filled up with a disk of ionised gas that shows similar kinematics. The conditions appear to be such that high Hi surface densities cannot build up because the $\mathrm{HI}_{\mathrm{i}}$ in the centre gets ionised. This could be connected to the fact that more luminous early-type galaxies often have a halo of hot gas, that could interact with the Hi and ionise it (e.g. Goudfrooij 1998). This could also explain the different excitation of the optical gas that is observed.

In a few low-luminosity galaxies, there is still evidence that the Hi has accreted recently, a process that appears to occur more often in more luminous galaxies. The different characteristics of the Hi in these galaxies suggest that the accretion of the Hi occurs in a different way in low-luminosity galaxies compared to the more luminous ones. In lowluminosity galaxies, acccretion appears to result in a more regular $\mathrm{HI}$ structure. This could also be due to interactions with a halo of hot gas playing a role in luminous galaxies. If Hi falls into a more luminous galaxy, the Hi could get partially ionised and may not have time to settle in a disk-like structure. In NGC 4696 such an interaction could be occurring (Sparks, Macchelfo \& Golombek 1989; de Jong et al. 1990), although this galaxy is in a cluster and it may not be representative for the galaxies we have studied.

Another factor affecting the way gas is accreted could be the environment. Several of the more luminous galaxies we studied are in small groups of galaxies, while the low-luminosity galaxies are more isolated. Interactions and accretions are of course more common in small groups and less relaxed Hi structures should be more common. Also the luminous galaxies with regular Hi structures tend to be more isolated, consistent with the idea that environment plays an important role in the evolution of $\mathrm{HI}$ in early-type galaxies.

\section{Acknowledgments}

The optical images shown in this paper are taken from the Digital Sky Survey. These images are based on photographic data obtained using the UK Schmidt Telescope. The UK Schmidt Telescope was operated by the Royal Observatory Edinburgh, with funding from the UK Science and Engineering Research Council, until 1988 June, and thereafter by the Anglo-Australian Observatory. Original plate material is copyright of the Royal Observatory Edinburgh and the Anglo-Australian Observatory. The plates were processed into the present compressed digital form with their permission. The Digitized Sky Survey was produced at the Space Telescope Science Institute under US Government grant NAG W-2166.

\section{References}

Bender, R. 1996, in New Light on Galaxy Evolution, IAU Symp. 171, ed. R. Bender \& R. Davies (Dordrecht: Kluwer), p. 181

Bregman, J. N., Hogg, D. E., \& Roberts, M. S. 1992, ApJ, 387,484

Canizares, C. R., Fabbiano, G., \& Trinchieri, G. 1987, ApJ, 312,503 
Davies, R. L., Efstathiou, G., Fall, S. M., Illingworth, G. D., \& Schechter, P. 1982, ApJ, 266, 41

de Jong, R., \& Davies, R. 1997, MNRAS, 285, 1

de Jong, T., Norgaard-Nielsen, H. U., Jorgensen, H. E., \& Hansen, L. 1990, A\&A, 232, 317

Goudfrooij, P. 1998, in Star Formation in Early-Type Galaxies, ed. P. Carral \& J. Cepa, ASP Conf. Proc., in press (astro-ph/980957)

Kauffmann, G. 1996, MNRAS, 281, 487

Knapp, G. 1998, in Star Formation in Early-Type Galaxies, ed. P. Carral \& J. Cepa, ASP Conf. Proc., in press (astro-ph/9808266)

Knapp, G. R., Turner, E. L., \& Cunniffe, P. E. 1985, AJ, 90,54

Lake, G., \& Schommer, R. A. 1984, ApJ, 280, 107

Lake, G., Schommer, R. A., \& van Gorkom, J. H. 1987, ApJ, 314, 57

Lauer, T. 1997, in Second Stromlo Symposium: The Nature of Elliptical Galaxies, ed. M. Arnaboldi, G. S. Da Costa \& P. Saha, ASP Conf. Ser. Vol. 116 (San Francisco: ASP), p. 113
Morganti, R., Sadler, E., Oosterloo, T., Pizzella, A., \& Bertola, F. 1997 a, AJ, 113, 937

Morganti, R., Sadler, E., \& Oosterloo, T. 1997b, in Second Stromlo Symposium: The Nature of Elliptical Galaxies, M. Arnaboldi, G. S. Da Costa \& P. Saha, ASP Conf. Ser. Vol 116 (San Francisco: ASP), p. 354

Morganti, R., Oosterloo, T., \& Tsvetanov, Z. 1998a, AJ, 115,915

Morganti, R., Oosterloo, T., Sadler, E. M., \& Vergani, D. 1998b, in Star Formation in Early-Type Galaxies, ed. P. Carral \& J. Cepa, ASP Conf. Ser. (San Francisco: ASP), in press (astro-ph/9809074)

Sadler, E. M. 1987, in Structure and Dynamics of Elliptical Galaxies, IAU Symp. 127, ed. T. de Zeeuw (Dordrecht: Kluwer), p. 125

Sparks, W. B., Macchetto, F., \& Golombek, D. 1989, ApJ, 345,153

Walsh, D. E. P., Van Gorkom, J. H., Bies, W. E., Katz, N., Knapp, G. R., \& Wallington, S. 1990, ApJ, 352, 532 